\title{
Dwarf Spheroidal Galaxies: Searching for Diffuse Emission in the Darkest Structures
}

\section{Marco Regis*}

Dipartimento di Fisica, Università di Torino and INFN, Torino, Italy

E-mail: regiseto.infn.it

\begin{abstract}
The dwarf spheroidal (dSph) satellites of the Milky Way are the darkest galaxies known in the Universe. They pose important challenges to our understanding of structure formation on small scales and provide an optimal laboratory for indirect searches of particle dark matter (DM). In this talk, I will present the first interferometric radio campaign devoted to the search of diffuse synchrotron emission in dSphs. It was conducted with the Australia Telescope Compact Array in the frequency band 1.1-3.1 GHz pointing to six Milky Way satellites. No significant evidence for an extended emission was found, and I will discuss the implications for non-thermal sources (including particle DM) in dSphs.
\end{abstract}

XI Multifrequency Behaviour of High Energy Cosmic Sources Workshop 25-30 May 2015

Palermo, Italy

\footnotetext{
* Speaker.
} 


\section{Introduction}

Dwarf spheroidal galaxies (dSph) are crucial objects for near-field cosmology. Their structure, chemical composition and kinematics pose important challenges to our understanding of galaxy formation (see, e.g., [1] for a recent review).

Recent searches for dwarf galaxies in the Sloan Digital Sky Survey data have more than doubled the number of known dSph satellite galaxies of the Milky Way (MW), and have revealed a population of ultra-faint galaxies, less luminous than any galaxy previously known [1]. In the last decade, twenty five new dwarf galaxy companions of the MW and M31 have been discovered. Within the first year of operation, the Dark Energy Survey (DES) has just found nine new dSph candidates $[2,3]$. Survey completeness studies suggest that a few hundreds of these extremely faint MW satellites should be discovered in the near future.

Spectroscopic studies have revealed that the recently discovered dSphs are the faintest (the most extreme ultra-faint dwarfs have luminosities smaller than the average globular cluster $L_{V} \sim$ $\left.10^{3}-10^{4} L_{\odot}\right)$, most dark matter $(\mathrm{DM})$ dominated, and most metal poor galaxies in the Universe (with mean stellar metallicity $\langle[\mathrm{Fe} / \mathrm{H}]\rangle \sim-2$ ).

$\mathrm{dSph}$ galaxies have been also recognized as optimal laboratories for indirect DM searches [4]. Due to their proximity, high DM content, and low level of astrophysical backgrounds, dSphs are widely considered to be among the most promising targets for detecting the diffuse electromagnetic radiation possibly induced by DM annihilations or decays.
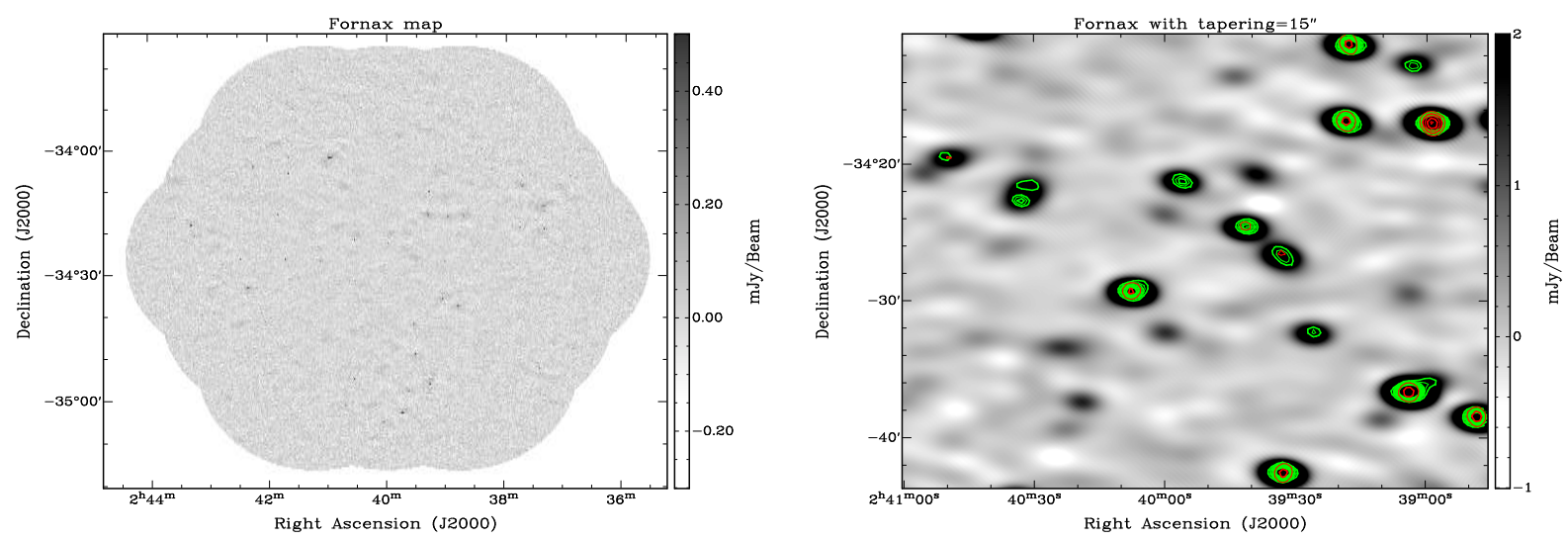

Figure 1: Left panel: Grayscale of the observational mosaic map after data reduction for the Fornax field of view. Right panel: Central region of the Fornax observational map after applying a Gaussian taper of 15 arcsec of FWHM. Contours from NVSS (from $2 \mathrm{mJy}$, green) and SUMSS (from $10 \mathrm{mJy}$, red) sources are overlaid. Plots are taken from [5].

\section{Observations and Data Analysis}

The observations presented in this talk were performed during July/August 2011 with the six 22-m diameter ATCA antennae operating in the frequency range 1.1-3.1 GHz. The targets included three classical dSphs (CDS), Carina, Fornax, and Sculptor, and three ultra-faint dSphs (UDS), BootesII, Hercules and Segue2, for a total of 123 hours of observing time. The array configuration was formed by a core of five antennae with maximum baseline of about $200 \mathrm{~m}$, and a sixth antenna 

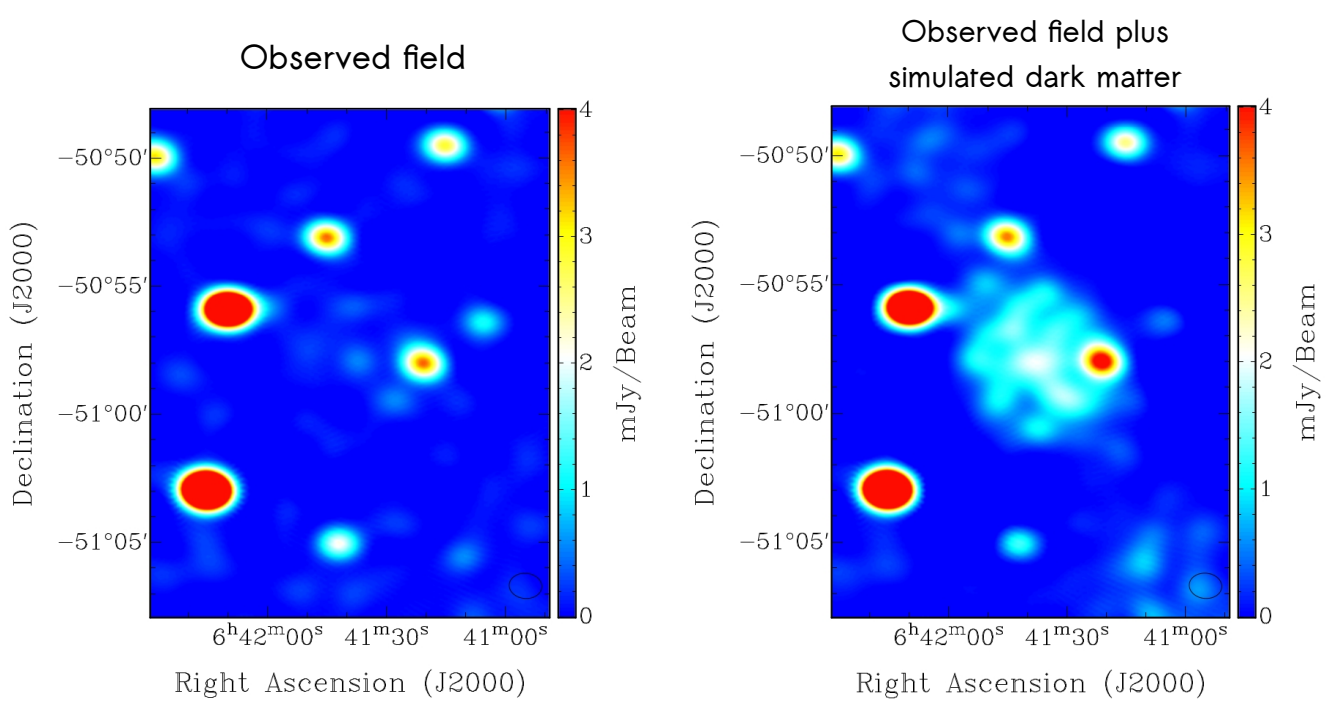

Figure 2: The observed Carina field (left) and how it would have looked like in presence of a bright DM signal (right). For the DM signal, we choose an example with DM particle mass of $100 \mathrm{GeV}$, annihilation into $b \bar{b}$ with a rate of $\left\langle\sigma_{a} v\right\rangle=10^{-25} \mathrm{~cm}^{3} \mathrm{~s}^{-1}$, and DM density as derived from dynamical measurements (see PaperIII) with a Burkert spatial profile. We set the magnetic field to $B_{0}=1 \mu \mathrm{G}$ and neglect spatial diffusion of electrons and positrons.

located at about $4.5 \mathrm{~km}$ from the core. The data were reduced using the Miriad data reduction package [6], following a standard calibration scheme. Details about the observing setup and data reduction are provided in [5] (Paper I).

In the imaging process, we produced two types of maps. ${ }^{1}$ First, data were imaged in highresolution maps (where the short baselines of the core are down-weighted) which can probe scales from few arcsec to few tens of arcsec, with rms noise of 30-40 $\mu \mathrm{Jy}$. In the second set of maps, we apply a Gaussian taper of 15 arcseconds (which down-weighs long baselines involving the sixth antennae) to the data before Fourier inversion. The synthesized beam of these maps becomes about 1 arcmin, and the largest scale which can be well imaged is around 15 arcmin. Because of confusion limitation, the rms noise raises up to 0.1-0.15 mJy. The main properties of the maps are summarized in Table 1 of [7] (Paper II). An example is reported in Fig. 1.

The tapered maps have a better sensitivity for testing the presence of a truly diffuse emission on scales above few arcmin, because of their larger beam. For these maps, point sources (i.e., discrete sources in the high resolution maps) are a "background" that can be subtracted. The discrete sources are mostly AGNs, as discussed in Paper I, where we constructed a catalogue. We perform the subtraction of sources before Fourier inversion (namely, in the visibility plane) with the CLEAN component of the corresponding high-resolution map used as the input source model. This procedure is described in Sec. 3.1.1 of Paper II, and allows to bring the rms of the tapered map down by a factor of few (see Fig. 5 of Paper II). To understand the impact of different imaging/subtraction methods on the intensity bounds, see also Fig. 6 in Paper II.

\footnotetext{
${ }^{1}$ Maps and source catalogue of this project can be retrieved at http://personalpages.to.infn.it/ regis/c2499.html.
} 

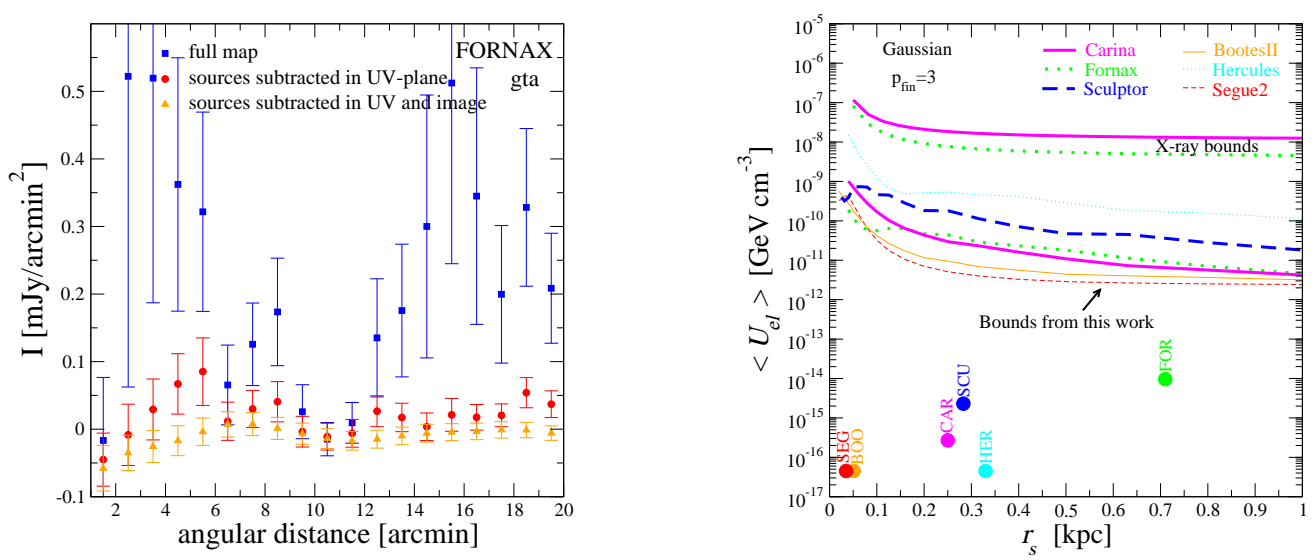

Figure 3: Left panel: Radial distribution of measured emission in the Fornax map after applying a Gaussian taper with FWHM=15". The emission is averaged in spherical annuli of 1 arcmin, and shown as a function of the distance from the center. Right panel: 95\% C.L. upper bounds on spatial average $\left\langle U_{e l}\right\rangle=3 r_{s}^{-3} \int_{0}^{r_{s}} d r r^{2} U_{e l}\left(r / r_{s}\right)$ versus spatial extension $r_{s} . U_{e l}(r)$ is the total equilibrium distribution of CR electrons and positrons in the dSph: $U_{e l}(r)=\int_{0.1 \mathrm{GeV}}^{1 \mathrm{TeV}} d E E n_{e}(E, r)$. For $n_{e}$, a power law energy spectrum with $p_{\text {fin }}=3$ and a Gaussian spatial profile are assumed. The upper lines show the corresponding X-ray bounds for the Carina and Fornax dSphs obtained following [8]. Circles show the expected CR density from the dSph SFR reported in Paper II. Plots are taken from [7].

\section{Discussion}

In Fig. 3a, we show the radial distribution of the observed surface brightness (in the example of Fornax). The points are the average of the emission in spherical annuli of width of 1 arcmin, as a function of the distance from the $\mathrm{dSph}$ center. Blue squares include sources and the emission is not compatible with a null signal. Red circles show the case with sources subtracted in the visibility plane. In some cases, they show an evidence of emission. However, the pattern is always similar to the case including sources, although with a much lower amplitude. This suggests that it is not a truly extended emission but rather a residuals of subtraction. This interpretation is supported also by the fact that after masking the region occupied by sources in the original map, we found that the curves do not show statistically significant deviations from the zero level. Orange triangles show instead the case with sources subtracted both in the visibility and image planes. They are always compatible with a null signal. Therefore, we do not obtain any significant evidence of a diffuse (above arcmin scale) emission in the dSph fields.

Fig. 2 shows an example of the observed fields (left) and how it would have looked like in presence of a bright DM diffuse signal (right). The simulated map is obtained by Fourier transforming the theoretical DM and adding the outcome to the actual data in the visibility plane.

We now discuss the impact of our non-detection on dSph properties. In Fig. 3b, we present constraints for the spatially averaged equilibrium distribution $\left\langle U_{e l}\right\rangle$ of cosmic-ray (CR) electrons and positrons in the $\mathrm{dSph}$. The bounds are compared to estimates derived from late-time star formation (SF) in $\mathrm{dSph}$ reported in Paper II. Assuming these estimates are realistic, we need a few 
order-of-magnitude improvement in the observational sensitivity (i.e., to reach a fraction of $\mu \mathrm{Jy}$ to $\mathrm{nJy}$ ), to probe the CDS emission, which is thus possibly achievable by the SKA. The emission related to SF in UDS would require a very high sensitivity even for the SKA.

In Fig. 4, we show bounds on the WIMP annihilation/decay rate as a function of the mass for leptonic (left) and hadronic (right, red curves) final states of annihilation/decay, derived in [9] (Paper III). They are comparable to the best limits obtained with gamma-ray observations and are much more constraining than what obtained in the X-ray band or with previous radio observations. In Paper II and III, we described the associated uncertainties, which are mostly given by the shape of the DM profile and the dSph magnetic properties, defining optimistic (OPT), average (AVE) and pessimistic (PES) scenarios. Since dSphs are poorly known systems, large uncertainties are present in the predicted signal. On the other hand, the SKA and its precursor will be able to progressively probe a signal from WIMP scenarios with "thermal" annihilation rate and masses up to few TeV, irrespective of astrophysical assumptions, as shown in Fig. 4 (blue curves, see also [12]).

With this pilot project, we demonstrated that radio interferometric observations are a suitable strategy to search for a WIMP-induced diffuse emission in dSphs. The dramatic advancements of radio telescopes we will witness in the forthcoming years offer the possibility to close in on the WIMP parameter space.
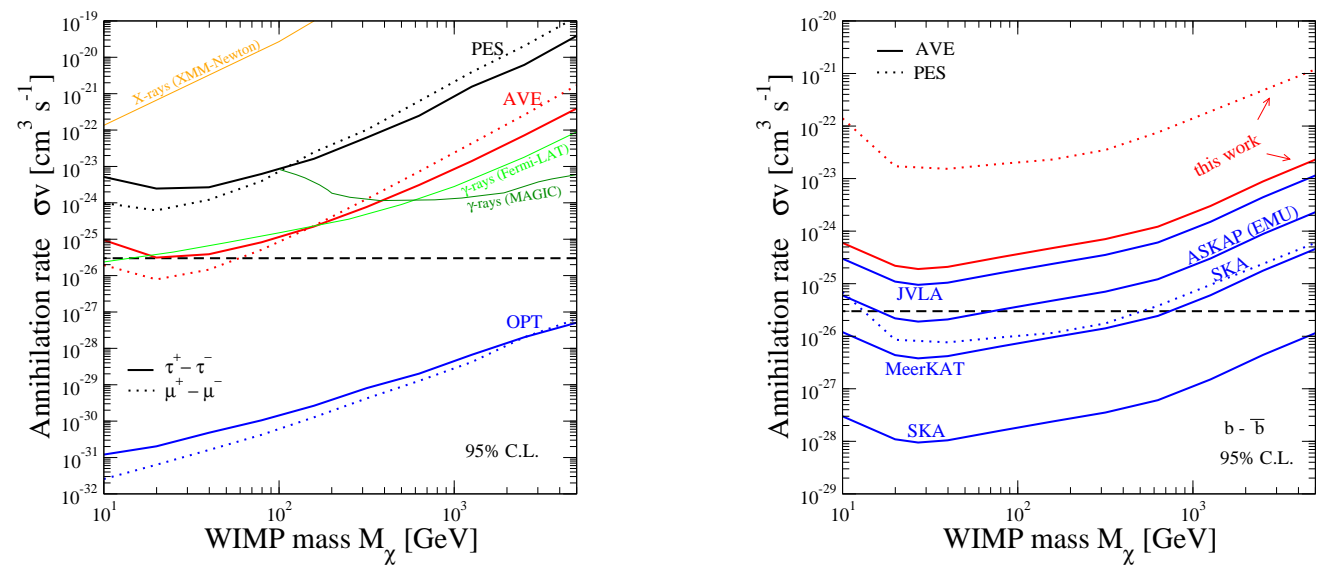

Figure 4: $95 \%$ C.L. upper bounds on the velocity averaged annihilation cross-section as a function of the WIMP mass. Left panel: Annihilations into $\tau^{+}-\tau^{-}$(solid) and $\mu^{+}-\mu^{-}$(dotted) for the scenarios OPT (blue), AVE (red), and PES (black) described in Paper III. We show also the bounds from the analysis of dSphs in Refs. [10] (light-green) and [11] (dark-green) at gamma-ray frequencies, and in Ref. [8] (orange, AVE scenario) in the X-ray band, for the $\tau^{+}-\tau^{-}$finale state. Right panel: Prospects of detection for WIMPs annihilating into $b-\bar{b}$ in the AVE and PES scenarios. See Paper III for details about models and experimental configurations. Plots are taken from [9].

\section{References}

[1] A. W. McConnachie, The observed properties of dwarf galaxies in and around the Local Group, Astron. J. 144 (2012) 4 [arXiv:1204.1562 [astro-ph.CO]]. 
[2] S. E. Koposov, V. Belokurov, G. Torrealba, \& N. W. Evans, Beasts of the Southern Wild : Discovery of nine Ultra Faint satellites in the vicinity of the Magellanic Clouds, Astrophys. J. 805 (2015) 130.

[3] The DES Collaboration 2015, Eight New Milky Way Companions Discovered in First-Year Dark Energy Survey Data, arXiv:1503.02584.

[4] S. Colafrancesco, S. Profumo and P. Ullio, Detecting dark matter WIMPs in the Draco dwarf: a multi-wavelength perspective, Phys. Rev. D 75 (2007) 023513 [arXiv:astro-ph/0607073].

[5] M. Regis, L. Richter, S. Colafrancesco, M. Massardi, W. J. G. de Blok, S. Profumo and N. Orford, Local Group dSph radio survey with ATCA (I): Observations and background sources, Mon. Not. Roy. Astron. Soc. 448 (2015) 3731 [arXiv:1407.5479 [astro-ph.GA]] (Paper I).

[6] R. J. Sault, P. J. Teuben and M. C. H. Wright, Astronomical Data Analysis Software and Systems IV 77 (1995) 433.

[7] M. Regis, L. Richter, S. Colafrancesco, S. Profumo, W. J. G. de Blok and M. Massardi, Local Group dSph radio survey with ATCA (II): Non-thermal diffuse emission, Mon. Not. Roy. Astron. Soc. 448 (2015) 3747 [arXiv:1407.5482 [astro-ph.GA]] (Paper II).

[8] T. E. Jeltema and S. Profumo, Searching for Dark Matter with X-ray Observations of Local Dwarf Galaxies, arXiv:0805.1054 [astro-ph].

[9] M. Regis, S. Colafrancesco, S. Profumo, W. J. G. de Blok, M. Massardi and L. Richter, Local Group dSph radio survey with ATCA (III): Constraints on Particle Dark Matter, JCAP 1410 (2014) 016 [arXiv:1407.4948 [astro-ph.CO]] (Paper III).

[10] M. Ackermann et al. [Fermi-LAT Collaboration], Dark Matter Constraints from Observations of 25 Milky Way Satellite Galaxies with the Fermi Large Area Telescope, Phys. Rev. D 89 (2014) 042001 [arXiv:1310.0828 [astro-ph.HE]].

[11] J. Aleksic et al., Optimized dark matter searches in deep observations of Segue 1 with MAGIC, JCAP 1402 (2014) 008 [arXiv:1312.1535 [hep-ph]].

[12] S. Colafrancesco, M. Regis, P. Marchegiani, G. Beck, R. Beck, H. Zechlin, A. Lobanov and D. Horns, Unveiling the nature of Dark Matter with the SKA,

Chapter of SKA Science Book (2014) [arXiv:1502.03738 [astro-ph.HE]].

\section{DISCUSSION}

PETER BIERMANN: Can you use background polarized sources to check on Faraday rotation by the ISM in these dwarf galaxies?

MARCO REGIS: With current radio telescopes the detection of Faraday rotation is out of reach (because of the low thermal density and small size of dSphs). The corresponding bounds do not translate into competitive bounds for the magnetic field. It is however something which could be explored with the SKA. 